NS32-P03

\section{The impact of stereogenic center on the architecture of the water framework: 2-methylpyrrolidine and sec-butylamine hydrates}

Patryk Rzepiński ${ }^{1}$, Łukasz Dobrzycki ${ }^{1}$, Michał Cyrański ${ }^{1}$, Roland Boese

1. Chemistry Department, University of Warsaw, Warsaw, Poland email: przepinski@chem.uw.edu.pl

sec-Butylamine and 2-methylpryrrolidine are primary aliphatic and secondary cyclic amines, respectively. They can exist in enantiopure form or as a racemic mixture. In both cases they can interact with water molecules forming hydrates of rather differentiated architecture. Their analyses shed light on the influence of stereogenic center on crystal packing and, in consequence, on the transfer of chirality to water frameworks and on their complexity.

Using the in situ crystallization technique [1] assisted by IR laser focused radiation we were able to obtain crystals of racemic amines, (R)-2-methylpyrrolidine and (S)-sec-butylamine. For each case we obtained also hydrates. As expected, racemic 2-methylpyrrolidine or sec-butylamine crystallize in centrosymmetric group (monoclinic $P 2_{1} / n$ or orthorhombic Pccn, respectively), whereas (R)-2-methylpyrrolidine and (S)-sec-butylamine in non-centrosymmetric ones $\left(P 2_{1}\right.$, or $P 2_{1}{ }_{1} 2$, respectively). In the case of hydrates the amine molecules are incorporated to $3 \mathrm{D}$ water network. Racemic mixture and $\mathrm{R}$ isomer of 2-methylpyrrolidine lead to 7 hydrate but they differ in crystallographic systems ( $P 2_{1} / n$ or $P 2_{1} 2_{1} 2_{1}$, respectively). The case of $s e c$-butylamine is different because the presence of stereogenic center dramatically changes the symmetry. Racemic mixture of sec-butylamine and water leads to $7 \frac{1}{3}$ hydrate $\left(P 2_{1} / c\right.$ space group) while the mixture of $\mathrm{S}$ isomer and water gives 6 hydrate $\left(P 2_{1} 2_{1} 2_{1}\right.$ space group)

It is worth to mention that hydrogen atoms for 2-methylpyrrolidine hydrate (both racemic and enantiopure) are ordered whereas in the case of sec-butylamine hydrate they are disordered.

Acknowledgements The work has been supported by the National Science Center grant (NCN 2011/03/B/ ST4/02591)

References:

[1] Boese, R. (2014) Z. Krystallogr., 229, 595

Keywords: chirality, hydrates, in situ crystallization
MS32-P04

\section{Porous supramolecular architectures based on $\pi$-stacking interactions between discrete metal-adenine entities and theobromine/ caffeine methylxanthines}

Jon Pascual Colino ${ }^{1}$, Maite Perfecto-Irigaray ${ }^{1}$, Garikoitz Beobide Pacheco ${ }^{1}$, Oscar Castillo Garcia ${ }^{1}$, Antonio Luque Arrebola ${ }^{1}$, Sonia Pérez-Yañez ${ }^{1}$

1. Department of Inorganic Chemistry, University of the Basque Country, UPV/EHU, 01006 Vitoria-Gasteiz, Spain

email: jonpascu2@gmail.com

Taking into account the great potential of MOFs, this work is focused on a relatively recent new class of analogous porous materials in which coordination bonds are released from guiding the crystal structure and supramolecular interactions play this role. ${ }^{1}$ Herein we present the results obtained for $\pi-\pi$ stacking interactions as the driving force to develop porous supramolecular metal-organic frameworks (SMOFs) as an alternative to more directional hydrogen bonding interactions. In particular, the use of adenine and methylxanthines has given rise to several compounds presented in this communication. The blue compound $\left[\mathrm{Cu}_{7}(\mu \text {-ade })_{6}\left(\mu_{3}-\right.\right.$ $\left.\mathrm{OH})_{6}\left(\mu-\mathrm{H}_{2} \mathrm{O}\right)_{6}\right]$ (theo $)_{2} \cdot 28 \mathrm{H}_{2} \mathrm{O}$ (1) (ade: adeninato, theo: theobrominato) is formed by wheel-shaped cationic units where the $\mathrm{Cu}$ (II) atoms are bridged by hydroxide anions, water molecules, and adeninato ligands with a m- $\mathrm{NN} 3: \kappa \mathrm{N} 9$ coordination mode. The supramolecular assembly (Figure 1) takes place mainly through $\pi-\pi$ stacking interactions involving the adeninato ligands of the rigid heptameric entities and theobrominate moieties. Compound $\mathbf{1}$ exhibits an open-framework with voids representing $37 \%$ of the unit cell volume, but the plasticity of the $\pi-\pi$ interactions causes a reversible shrinkage of the porous system upon activation that precludes the adsorption of gas molecules.

Dark purple compounds $\left[\mathrm{Cu}_{2}(\mu \text {-ade })_{4}\left(\mathrm{H}_{2} \mathrm{O}\right)_{2}\right] \cdot 3 \mathrm{H}-$ theo $\cdot 7 \mathrm{H}_{2} \mathrm{O}(2),\left[\mathrm{Cu}_{2}(\mu \text {-ade })_{4}\left(\mathrm{H}_{2} \mathrm{O}\right)_{2}\right] \cdot 2 \mathrm{H}$ theo $\cdot 18 \mathrm{H}_{2} \mathrm{O}(\mathbf{3})$ and $\left[\mathrm{Cu}_{2}(\mu \text {-ade })_{4}\left(\mathrm{H}_{2} \mathrm{O}\right)_{2}\right] \cdot$ (caf) $\sim 6 \mathrm{H}_{2} \mathrm{O}$ (4) (Htheo: theobromine, caf: caffeine) contain neutral windmill units in which two copper atoms are bridged by four $\mathrm{m}-\kappa \mathrm{N} 3: \kappa \mathrm{N} 9$ adeninato ligands. Their crystal structures highly depend on the supramolecular interactions of the theobromine and caffeine bases. In compound $\mathbf{2}$, two theobromine molecules are hydrogen bonded to the Hoogsteen face of two trans-arranged adeninato ligands, whereas a third theobromine molecule is joined to the Watson-Crick face of one of the previous adeninato ligand. In compound $\mathbf{3}$, with a lower amount of theobromine, the Watson-Crick interaction is not present. In both compounds, the $3 \mathrm{D}$ crystal structure requires the additional presence of $\pi-\pi$ stacks between the theobromine molecules.

In compound $\mathbf{4}$, as the methyl groups of the caffeine molecule do not allow hydrogen bond interactions, the adeninato ligands are hydrogen bonded among them to generate, together with $\pi$-stacking interactions, supramolecular sheets containing rectangular windows in which the caffeine molecules are located. Only compound $\mathbf{4}$ showed permanent porosity, adsorbing a significant amount of $\mathrm{CO}_{2}(0.88 \mathrm{mmol}$ of $\mathrm{CO}_{2} / \mathrm{g}$ at 5 bar and $273 \mathrm{~K}$ ). The magnetic characterization of the compounds indicate a ferromagnetic behaviour for 
$\mathbf{1}$ and strong intradimeric antiferromagnetic interactions in compounds $\mathbf{2}$ and $\mathbf{4}$.

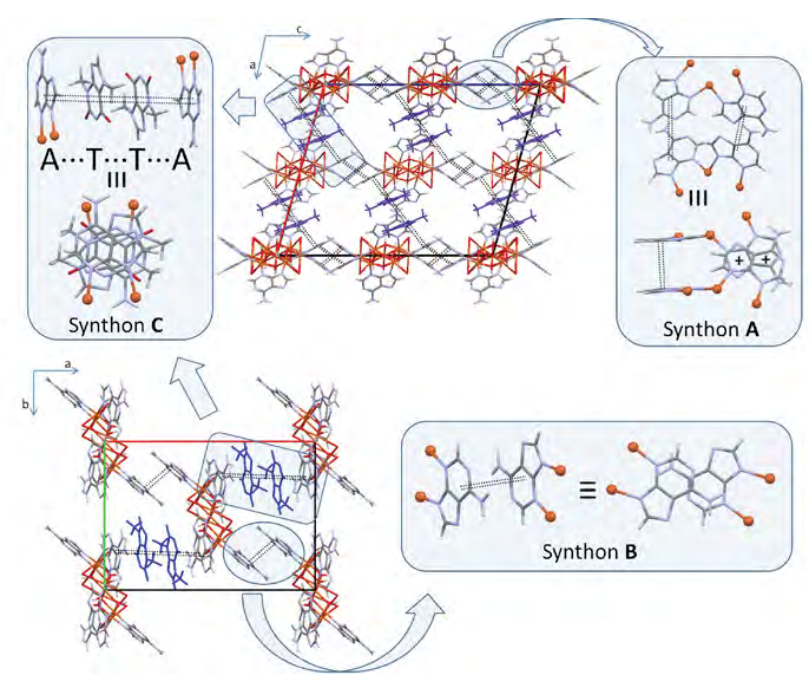

References:

[1] (a) Thomas-Gipson, J.; Pérez-Aguirre, R.; Beobide, G.; Castillo, O.; Luque, A.; Pérez-Yáñez, S.; Román, P.; (2015) Cryst. Growth Des., 15, 975-983. (b) Beobide, G.; Castillo, O.; Luque, A.; PérezYáñez, S. (2015) CrystEngComm, 17, 3051-3059.

Keywords: SMOFs, supramolecular interactions, $\pi-\pi$ stacking

\section{MS32-P06}

\section{Temperature-dependent supramolecular isomerism of lutetium-aminoterephthalate metal-organic frameworks: synthesis, crystallography and physical properties}

Alla Dikhtiarenko', Pablo Serra-Crespo ${ }^{2}$, Sonia Castellanos ${ }^{3}$, Alexey Pustovarenko ${ }^{4}$, Rafael Mendoza-Meroño ${ }^{5}$, Santiago García-Granda ${ }^{5}$, Jorge Gascon ${ }^{1}$

1. Kaust Catalysis Center, King Abdullah University of Science and Technology, Thuwal, Saudi Arabia

2. Radiation and Isotopes for Health, Department of Radiation Science and Technology, Faculty of Applied Sciences, Technical University Delft, Delft, Netherlands (Holland, Europe)

3. Advanced Research Center for Nanolithography, Amsterdam, Netherlands (Holland, Europe)

4. Chemical Engineering Department, Delft University of Technology, Delft, Netherlands (Holland, Europe)

5. Departamento de Química Física y Analítica, Universidad de Oviedo, Oviedo, Spain

email: alla.dikhtiarenko@kaust.edu.sa

Polymorphism or supramolecular isomerism is becoming an increasingly important subject in the field of crystal engineering, as it may facilitate the design of porous polymeric materials with targeted physical properties. ${ }^{[1]}$ In this context, the unique properties of MOF polymorphs, displaying both different crystal structures and identical molecular composition, allow for the derivation of structure-property relationships solely based on structural parameters or topology of the polymeric network. There are various key factors governing the formation of MOFs' polymorphic forms, including reaction time, temperature, concentration of precursors, $\mathrm{pH}$, solvent, modulators, and so on. Although numerous individual factors ${ }^{[2]}$ or their conjunction ${ }^{[3]}$ affect the occurrence of polymorphic forms, temperature is the one of the most important variables. The study of polymorphism is not only important in producing novel materials with target properties but may also be helpful in developing a fundamental understanding of the factors influencing crystal growth, such as reaction temperature.

In this line, we present the investigation on the relationship between the crystal structures and gas adsorption / fluorescence properties of lutetium(III) 2-aminoterephthalate MOFs isomorph series. Three supramolecular isomers of lutetium metal-organic framework, $\left\{\mathrm{Lu}_{2}\left(\mathrm{H}_{2} \mathrm{O}\right)_{4}(\mathrm{ATA})_{3} \cdot 4 \mathrm{H}_{2} \mathrm{O}\right\}$ n (Lu-ATA@RT), $\quad\left\{\mathrm{Lu}_{2}\left(\mathrm{H}_{2} \mathrm{O}\right)_{2}\left(\mathrm{C}_{3} \mathrm{H}_{7} \mathrm{NO}\right)_{2}(\mathrm{ATA})_{3}\right\}_{\mathrm{n}} \quad(\mathrm{Lu}-$ ATA@100) and $\left\{\mathrm{Lu}_{2}\left(\mathrm{C}_{3} \mathrm{H}_{7} \mathrm{NO}\right)(\mathrm{ATA})_{3}\right\}_{\mathrm{n}}$ (Lu-ATA@150), have been obtained from the reaction of $\mathrm{Lu}\left(\mathrm{NO}_{3}\right)_{3} \cdot 6 \mathrm{H}_{2} \mathrm{O}$ with 2-aminoterephthalic acid (ATA) at different temperatures. The resulting structures of Lu-ATA MOFs depend on the temperature applied during the synthesis, revealing a temperature-susceptible supramolecular isomerism. Single-crystal X-ray diffraction analyses suggest that new compounds with formula $\left\{\mathrm{Lu}_{2}(S)_{\mathrm{x}}(\mathrm{ATA})_{3}\right\}_{\mathrm{n}}(S=$ solvent: $\mathrm{H}_{2} \mathrm{O}$, DMF) display different three-dimensional architectures which consist on dinuclear lutetium building units. The supramolecular isomer Lu-ATA@RT, formed at room temperature, has a $p c u$-net topology while its double interpenetrated analogue, Lu-ATA@100, assembles at $100{ }^{\circ} \mathrm{C}$ under hydrothermal conditions. Hydrothermal synthesis at $150{ }^{\circ} \mathrm{C}$ affords formation of the dense Lu-ATA@150 cage- 